(CASE REPORT)

\title{
Generalized onset seizures during COVID-19 infection: Does it mean the diagnosis Epilepsy
}

\author{
Ekaterina Ivanova Viteva *
}

Medical University - Plovdiv, Bulgaria, Department of Neurology, UMHAT “St. George” - Plovdiv, Bulgaria.

Publication history: Received on 11 November 2020; revised on 17 November 2020; accepted on 18 November 2020

Article DOI: https://doi.org/10.30574/wjarr.2020.8.2.0419

\begin{abstract}
We present a case report of a 58-year old man who came to our Department of Neurology for a consultation 2 months after being diagnosed with COVID-19 infection. The patient had been febrile for a week and had had 2 generalized onset seizures with clonic jerks of the upper extremities in 2 consecutive days. Then he had been admitted at a department of infection diseases. COVID-19 PCR had been positive. EEG and CT scan of the brain had been absolutely normal. The patient had been treated with azithromycin for the COVID-19 infection and following a negative PCR test, he had gone home. During the hospital stay the patient had been diagnosed with Epilepsy and valproate treatment had been started. The patient did not accept the diagnosis and did not take the anticonvulsant regularly, moreover he was a truck driver and wanted to go back to work shortly. The control EEG showed no abnormalities and he had no more seizures. Therefore we did not accept the diagnosis Epilepsy and did not recommend a regular intake of antiepileptic medications.

In conclusion we would like to emphasize on the need of an individual approach to every patient. Provoked seizures which are symptoms of an infection do not necessarily mean a diagnosis Epilepsy and do not require chronic treatment, especially when they are not present after the acute stage and EEG is normal.
\end{abstract}

Keywords: COVID-19; Seizures; Epilepsy; Overdiagnosis; EEG

\section{Introduction}

The rapid global spreading of Coronavirus disease 2019 (COVID-19) is associated with the documentation of a variety of neurological symptoms. An increasing number of patients with seizures at the onset or in the course of the COVID-19 infection have been described recently. They may be a consequence of fever, hypoxia, metabolic derangements, organ failure or even cerebral damage such as encephalitis or encephalopathy [1-3]. The usual seizure types are motor (tonic, clonic or tonic-clonic) with or without loss of consciousness [4-6]. These clinical presentations set the question whether it is correct to diagnose the patients with epilepsy and start regular antiseizure therapy.

\section{Case report}

A 58-year old man, a truck driver, came to our Department of Neurology for a consultation 2 months after being diagnosed with COVID-19 infection. The patient had been febrile, with headache, sore throat for a week. He had been treated with clindamycin for 7 days without substantial improvement. Then while being febrile up to $39^{\circ} \mathrm{C}$, he had had a generalized onset seizure with bilateral tonic and clonic jerks of the upper extremities. The same seizure type had been observed in the next day. Then he had been admitted at a department of infection diseases. The full blood count and biochemistry had shown no abnormalities. COVID-19 PCR had been positive. The pulmonary X-ray had shown a small area of decreased aeration and a blurred contour of the diaphragm. EEG had visualized no pathological findings.

\footnotetext{
${ }^{*}$ Corresponding author: Ekaterina Viteva

Medical University - Plovdiv, Bulgaria, Department of Neurology, UMHAT "St. George" - Plovdiv, Bulgaria.
} 
The brain CT scan had been normal. During the hospital stay the patient had been febrile during the first 5 days, afterwards he had felt much better and had had no more seizures. He had been treated with azithromycin and paracetamol. The patient had been diagnosed with Epilepsy and Valproate treatment had been started. Following a negative PCR test in 10 days, the patient had gone home. He had been recommended to take valproate regularly, but the patient did not accept the diagnosis and did not take the anticonvulsant regularly. As he wanted to go back to work shortly, he came to consult about the diagnosis Epilepsy and the need for anticonvulsant treatment. The control EEG showed no abnormalities and he had no more seizures. Therefore we did not accept the diagnosis Epilepsy and did not recommend a regular intake of antiepileptic medications.

\section{Discussion}

The neurological complications of the novel COVID-19 infection and their underlying causes are not currently known at certain. However, according to the modern definition of Epilepsy, provoked seizures which are symptoms of an infection do not necessarily mean this diagnosis. Therefore, they do not require chronic treatment, especially when they are not present after the acute stage and EEG is normal. These patients certainly need long-term monitoring and regular clinical and EEG control. The overdiagnosis in our patient would mean a regular intake of an anticonvulsant, which could have adverse events and be associated with various drug interactions. Besides, this diagnosis will result in problems with his work and decreased quality of life.

\section{Conclusion}

The individual approach to every patient with seizures is very important. The overdiagnosis of Epilepsy should be avoided as it is associated with continuous treatment and may affect all aspects of patients' life.

\section{Compliance with ethical standards}

\section{Acknowledgments}

We have no acknowledgements to include.

\section{Disclosure of conflict of interest}

No conflicts of interest.

\section{Statement of informed consent}

Informed consent was obtained from the participant included in the study.

\section{References}

[1] Kuroda N. Epilepsy and COVID-19: Associations and important considerations. Epilepsy \& Behav. $2020 ; 108$ [107122].

[2] Asadi-Pooya A. Seizures associated with coronavirus infections. Seizure 2020; 79:49-52.

[3] French J, Brodie M, Caraballo R, Devinsky O, Ding D, Jehi L, Jette N, Kanner A, Modi A, Newton C, Patel A, Pennell P, Perucca E, Sander J, Scheffer I, Singh G, Williams E, Wilmshurst J, Cross H. Keeping people with epilepsy safe during the COVID-19 pandemic. Neurology 2020; 94:1-6.

[4] Moriguchi T, Harr N, Goto J, Harada D, Suqawara H, Takamino J, Ueno M, Sakata H, Kondo K, Myose N, Nakao A, Takeda M, Haro H, Suzuki-Inoue K, Kubokawa K, Oqihara S, Sasaki T, Kinouchi H, Kojin H, Ito M, Onishi H, Shimizu T, Sasaki Y, Enomoto N, Ishihara H, Furuya S, Yamamoto T, Shimada S. A first case of meningitis/encephalitis associated with SARS-Coronavirus-2, IJID 2020; 94:55-58.

[5] Fasano A, Cavallieri F, Canali E, Valzania F. First motor seizure as presenting symptom of SARS-CoV-2 infection. Neurol Sci 2020; 41:1651-1653.

Sohal S, Mansur M. COVID-19 presenting with seizures. IDCases 2020; 20:e00782. 